Research Article

\title{
Customer Behavior Analysis in Ticket Windows at Scenic Spots from Game-Theoretic Perspective
}

\author{
Peng Li, ${ }^{1}$ Tao Jiang $\mathbb{D},{ }^{2}$ Lu Liu, ${ }^{2}$ and Sherif I. Ammar ${ }^{3}$ \\ ${ }^{1}$ School of Tourism and Public Administration, Huzhou Vocational \& Technical College, Huzhou 313000, China \\ ${ }^{2}$ College of Economics and Management, Shandong University of Science and Technology, Qingdao 266590, China \\ ${ }^{3}$ Mathematics and Computer Science Department, Faculty of Science, Menoufia University, Menoufia 32511, Egypt
}

Correspondence should be addressed to Tao Jiang; jtao0728@163.com

Received 3 August 2021; Revised 9 October 2021; Accepted 27 October 2021; Published 8 November 2021

Academic Editor: Leonid Shaikhet

Copyright (c) 2021 Peng Li et al. This is an open access article distributed under the Creative Commons Attribution License, which permits unrestricted use, distribution, and reproduction in any medium, provided the original work is properly cited.

\begin{abstract}
In service industries, especially in some ticket windows at scenic spots, affected by congestion, some customers often find excuses or ask the acquaintances in the queue so as to jump the queue and complete their corresponding service as early as possible. In this paper, we model the queueing phenomenon in the ticket windows at scenic spots as a special queue, namely, the so-called "team queue." Although this phenomenon often happens in daily life, it is less well known to people. In a team queue, an arriving customer first searches the queue from the top to bottom to see if some of his teammates are already in the queue. If yes, he would join the queue and be served with his teammates; otherwise, he would join the queue at the tail. To this end, arising from the customers' perspective, the strategic behavior of queueing customers in the ticket windows at scenic spots is analyzed. On the basis of considering the waiting cost and reward, the individual strategies and social optimal strategies of queueing customers are derived, regarding the joining or balking dilemma and for the observable and unobservable cases. Finally, to demonstrate how various parameters affect the joining strategies, some numerical examples are provided.
\end{abstract}

\section{Introduction}

Nowadays, in the ticket windows at scenic spots, stations, and other places, customer service is basically on a firstcome-first-served (FCFS) discipline. However, because of the limitations of service capabilities and hardware facilities of service providers, a congestion phenomenon in queueing service frequently occurs; some queue-jumping customers often attempt to jump in queue or find excuses (ask the acquaintances in the queue) so as to cut in the queue and complete their corresponding service as early as possible. By jumping the queue, the queue-jumping customers can be served before customers who are already in line; as a result, the queueing phenomenon does not adhere to FCFS discipline and the act of jumping in the queue cannot be ignored in designing the service systems. On the basis of the queueing phenomenon in front of ticket windows at scenic spots, in this paper, the queueing phenomenon is modelled as a special queue, i.e., the team queue, which is less well known, although it occurs frequently in everyday life. In front of ticket windows at scenic spots, each customer in the waiting line belongs to a team. If a new arriving customer joins the team queue, he first searches the queue from the top to bottom to see if any of his acquaintances (teammates) are already in the waiting line; if yes, he accedes the queue and receives service together with his teammates; otherwise, he joins the queue at the tail.

In the existing literature about queue-jumping customers in the ticket windows, many studies mainly focused their analysis on obtaining the stationary distribution as well as key performance measures, such as He and Chavoushi [1], Boxma et al. [2], and Perel and Yechiali [3-6]. As far as we know, there seems to be insufficient research on the phenomenon of queueing service from the perspective of customers, and no research has analyzed the strategic behavior of queue-jumping customers in queueing systems. To this end, we will use the team queue to model the queueing service phenomenon in front of ticket windows at scenic 
spots, where the queue-jumping customers find their teammates in the queue to cut in, and analyze the team queue from an economic viewpoint with deriving the queuejumping customers' corresponding equilibrium strategies. The following are the benefits and contributions of this work.

1.1. Model. A queue-jumping customer is presented in a continuous-time team queue. This model is well suited to capturing the characteristics of customers lining up in front of the ticket windows at scenic spots.

1.2. Methodology and Results. From the viewpoint of queueing customers, queueing theory and game theory are used to examine the team queue. We create a queueinggame-theoretic model of a team queue and derive formulas for calculating customer strategies at different levels of information. By the elaborate successful formulation of the problem, the underlying paper expands the current theory of queueing economics and extends the theoretical literature of queueing theory and game theory.

1.3. Numerical Illustrations. This paper includes numerical examples to illustrate the effects of various parameters on customer strategies, as well as some qualitative take-away messages and intuitive explanations.

The outline structure of this paper is as follows: Section 2 contains a comprehensive overview of relevant studies. Section 3 describes the underlying team queue and constructs the reward-cost structure. The individual optimal strategies and the expected social welfare per unit of time in the observable case are investigated in Section 4 . The equilibrium joining strategies for the unobservable case are deduced in Section 5. Section 6 includes some numerical findings that demonstrate the effects of various parameters on customer strategic action. In Section 7, we wrap up the paper and summarize the key results of our paper.

\section{Literature Review}

The literature on the queueing model with queue-jumping customers dates back to Larson [7]. In [7], the author discussed the queueing system with queue-jumping behavior from the perspective of social justice and psychology. Later, Afèche and Mendelson [8] studied a queueing system in which customers' position in the queue depends on how much they pay. He and Chavoushi [1] investigated the queueing systems with queueing jumpers and derived the waiting time periods of two types of customers. Recently, Perel and Yechiali [3-6] and Jiang et al. [9-11] also considered the so-called "Israeli queue," which reflects a realworld situation and can be viewed as a queue-jumping phenomenon. Arriving customers in an Israeli queue first search for their friends or acquaintances already lining up in the waiting region, and they could be served before the customers who are already in the queue. As a result, the Israeli queue can model the queue-jumping phenomenon, which is also common in China. Concretely, the authors of
[3] looked at a polling scheme with an infinite number of queues, where all customers in a queue can be served at the same time by the server in that queue; i.e., at each queue, service is provided in batches of any size. In [4-6], the authors used different approaches to analyze the preemptive priority Israeli queue, the retrial Israeli queue, and the general group-joining policy of Israeli queue and obtained the stationary distribution as well as some primary performance measures. In addition, the authors considered the Israeli queue with multiple servers in [9], where the service rate of each server varies from low to high, depending on the number of groups in the queue. In [10], the authors considered a multiphase random environment Israeli queue, where two regions in front of the queue and the holding time of an external environment vary depending on the system's state. In [11], the tail asymptotics for the retrial Israeli queue was studied in depth, since the explicit closed solution for a stationary distribution is difficult to obtain while each retrial customer in the orbit is making independent and repeated attempts to accept the service. In all of the papers above, we discovered that the Israeli queue is modelled as follows: arriving customers could form groups, and service time at each group is independent of the group size. A new arriving customer first searches each group leader; if he knows a leader, he will join the group and be served together with all customers in this group. By using some effective methods, the authors mainly focused their analysis on obtaining the stationary distribution as well as key performance measures.

Our model also falls into the category of strategic customers. Some excellent references are studies by Naor [12], Edelson and Hildebrand [13], Hassin and Haviv [14], Hassin [15], Hassin and Roet-Green [16], Ibrahim [17], etc.

In reality, it is a new attempt to analyze the strategic behavior of customers in batch service queueing systems. For instance, Economou and Manou [18] have explored M/ $\mathrm{M} / 1$ queue in a substitutional environment where all existing customers will be served upon the expiration of service. Manou et al. [19] examined the strategic behavior of passengers at a transportation station, where all existing passengers can be excised as soon as the vehicle stops at the station. Customer-joining behavior in batch service queueing systems was also studied in [20], in which a fixed number of customers could be served at once. Then, Bountali and Economou [21] investigated the batch services model under imperfect information. Wang et al. [22] studied the strategic behavior of passengers in the queueing system with batch transfer under threshold policy and obtained the threshold joining strategies of passengers. Afimeimounga et al. [23], Chen et al. [24], and the references therein are recommended for the interested readers who want to know more about the customer behavior analysis in batch queueing systems.

\section{Model Description}

For the sake of analysis, for the queueing service phenomenon in front of some ticket windows at scenic spots, we assume that all queueing customers are queue jumpers; i.e., each arriving customer first finds his friend in the queue so 
as to cut in the queue and complete his corresponding service as early as possible. Therefore, the underlying queueing phenomenon can be modelled as an $M / M / 1$ "team queue (Israeli queue)." We use "he" to represent a customer and "team queue" to represent the underlying queueing system with queue-jumping customers for ease of exposition. More concretely, we also assume that customers arrive at the system according to a Poisson process with rate $\lambda$, and the service time, regardless of size (an unlimited size), follows an exponential distribution with mean $\mu^{-1}$. If a new arrival determines to join the system, he will first look for the group leader, and if he finds an acquainted leader, he will directly join the group and receive service alongside the group leader. Actually, the assumption is reasonable. In front of the ticket windows at scenic spots, the head of the group buys several tickets at a time. In today's information society, the influence of the number of tickets purchased on the service time can be ignored. We also suppose that the value of the probability that a new arrival is familiar with a group leader is $\theta$, namely, if $m$ groups are in the team queue, then with probability $(1-\theta)^{k-1} \theta, 1 \leq k \leq m$, an arriving customer who decides to join the team queue would be assigned to the $k$ th group or would create a new group with probability. We also suppose that a new arrival could also join the being-served group. From the model description, the service is no longer subject to FCFS and a new arrival could be served before the customers who are already in the line.

At time $t$, define $L(t)$ as the different number of groups; then, the state of team queue $\{L(t), t \geq 0\}$ could be described by a continuous-time Markov chain (CTMC). The state space of the team queue is $\Omega=\{0,1,2, \ldots\}$.

Unlike recent team queue papers, which concentrate on the performance analysis, in the current paper, we mainly discuss the team queue from the queueing customer perspective, where arriving customers are fully strategic, and they can choose whether to join the team queue or not, depending on their expected utility.

We construct a decision framework $U=R-C E[W]$, where $U$ is the net utility, $R$ is the valuation of service offerings, $C$ is the delay sensitivity, and $E[W]$ records the expected sojourn time (waiting and service time) of a joining customer. Then, depending on the net utility $U$, if $U \geq 0$, a newly arrived customer will join the queue, and vice versa.

Next, by distinguishing the observable and the unobservable cases, we study the strategic joining behavior of customers. Concretely, we have the following: (1) observable case: customers are allowed to know the number of groups in the team queue and cannot see in advance if they have friends in the queue; (2) unobservable case: customers are not allowed to know the number of groups in the team queue and cannot observe in advance if they have friends in the queue, but they know the value of parameter $\theta$.

\section{Observable Case Analysis}

We first examine the observable team queue. Customers know about the team queue when they arrive in the observable situation; i.e., they might be informed of the number of different groups in the queue but cannot see in advance if they have friends in the queue. Next, we provide a lemma to show the expected utility of a new arriving customer when he arrives to the system.

Lemma 1. For the observable team queue, if a new arrival discovers that $n$ groups exist and he decides to join the queue, then the utility is

$$
U_{\text {obs }}(n)=R-\frac{C}{\mu} \frac{1-(1-\theta)^{n+1}}{\theta} .
$$

Proof. First, assume that a new arrival discovers the team queue is empty upon his arrival and decides to join the team queue; he will create a group and get served immediately by the server; hence, his sojourn time is equal to $1 / \mu$. Next, we consider that a new arrival who discovers that $n>0$ groups exist upon his arrival and determines to join the queue. We consider two scenarios that whether the customer knows the leaders of present groups or not.

Case 1. The arriving customer knows the leaders of present groups. In this case, if $n>0$ groups exist, then the probability that the new arrival knows the leader of the $k$ th group is $(1-\theta)^{k-1} \theta$, and if he joins the $k$ th group, his sojourn time is $k / \mu, 1 \leq k \leq n$.

Case 2. The arriving customer does not know any leaders of all present groups. In this case, the probability that the new arrival forms a new group is $(1-\theta)^{n}$ and his sojourn time is $(n+1) / \mu$.

According to the decision framework, if an incoming customer discovers that $n$ groups exist in the team queue and determines to join the queue, the utility would be

$$
U_{\mathrm{obs}}(n)=R-C E\left[W_{n}\right]=R-C\left(\frac{\theta}{\mu}+\frac{2 \theta(1-\theta)}{\mu}+\cdots+\frac{n \theta(1-\theta)^{n-1}}{\mu}+\frac{(n+1)(1-\theta)^{n}}{\mu}\right),
$$


where $W_{n}$ records the expected sojourn time of the new arrival when he notices that $n \geq 1$ groups exist in the team queue. After some calculations, we have

$$
U_{\text {obs }}(n)=R-\frac{C}{\mu} \frac{1-(1-\theta)^{n+1}}{\theta} .
$$

Following that, we could locate the unique individual optimal pure strategy by using Lemma 1 .

Theorem 1. For the observable model, we could obtain the unique individual optimal pure strategy:

Scenario 1. If $R<(C / \mu)$, balking is the unique individual optimal pure strategy.

Scenario 2. If $(C / \mu) \leq R<(C / \mu \theta)$, we could obtain the unique threshold strategy: if a new arrival discovers that $n$ groups exist in the team queue and $n \leq n_{e}$, he would join the team queue; otherwise, he would balk, where $n_{e}\left(=\left\lfloor n^{*}\right\rfloor\right)$ and

$$
n^{*}=\frac{\ln K}{\ln (1-\theta)}-1
$$

with $K=(C-R \mu \theta) / C$.

Case 3. If $R \geq(C / \mu \theta)$, joining is the unique individual optimal pure strategy.

Proof. Differentiating $U_{\text {obs }}(n)$ about $n$, we have

$$
\frac{\mathrm{d} U_{\mathrm{obs}}(n)}{\mathrm{d} n}=\frac{C}{\mu \theta}(1-\theta)^{n+1} \ln (1-\theta)<0 .
$$

Then, $U_{\text {obs }}(n)$ is monotone decreasing with respect to $n$; as a result, if $U_{\text {obs }}(n)<0$, a new arrival would balk; otherwise, he is more willing to join the team queue. Next, we consider three cases to obtain the results:

When $R<(C / \mu)$, we have $U_{\text {obs }}(0)<0$. In this case, balking is the unique individual optimal strategy.

When $(C / \mu) \leq R<(C / \mu \theta)$, we have $U_{\text {obs }}(0) \geq 0$ and $U_{\text {obs }}(+\infty)<0$. In this case, there is a unique root $n^{*}$ that satisfies $U_{\text {obs }}(n)=0$. According to the equation, we have $n^{*}=(\ln K / \ln (1-\theta))-1$ with $K=(C-R \mu \theta) / C$. Since $U_{\text {obs }}(n)$ is monotone decreasing with respect to $n$, a new arrival would join the team queue when $n \leq n_{e}\left(=\left\lfloor n^{*}\right\rfloor\right)$; otherwise, he would balk the queue.

When $R \geq(C / \mu \theta)$, we have $U_{\text {obs }}(n) \geq 0$. Therefore, in this case, joining is the unique individual optimal strategy.

Now, we look at the issue from the viewpoint of a social planner's perspective; i.e., by constructing a social welfare function, we could get the optimum threshold value to maximize the social welfare.

Theorem 2. For the observable model, if all new arrivals adopt a threshold strategy (the threshold value $n_{s}$ ), we then have the expected net benefit, which has the following form:

$$
S_{\mathrm{obs}}^{\mathrm{soc}}\left(n_{s}\right)=\lambda\left(n_{s}\right) R-\mathrm{CE}\left[L_{\mathrm{obs}}\right] \text {, }
$$

where

$$
\begin{aligned}
\lambda\left(n_{s}\right) & =\lambda\left(1-\frac{(\lambda / \mu)^{n_{s}+1}(1-\theta)^{n_{s}\left(n_{s}+1\right) / 2}}{\sum_{n=0}^{n_{s}+1}(\lambda / \mu)^{n}(1-\theta)^{n(n-1) / 2}}\right), \\
E\left[L_{\mathrm{obs}}\right] & =\lambda\left(n_{s}\right) \sum_{n=0}^{n_{s}} \frac{(\lambda / \mu)^{n}(1-\theta)^{n(n-1) / 2}}{\sum_{k=0}^{n_{s}+1}(\lambda / \mu)^{k}(1-\theta)^{k(k-1) / 2}} \frac{1-(1-\theta)^{n+1}}{\mu \theta} .
\end{aligned}
$$

Proof. First, in the observable case, denote by $\pi_{\mathrm{obs}}^{\text {soc }}(n)$ its corresponding steady-state distribution, i.e., the probability that $n$ groups exist in the team queue; then, under the threshold strategy with threshold value $n_{s}$, the distribution can be obtained by solving the following balance equations:

$$
\begin{aligned}
\lambda \pi_{\mathrm{obs}}^{\mathrm{soc}}(0) & =\mu \pi_{\mathrm{obs}}^{\mathrm{soc}}(1), \\
{\left[\lambda(1-\theta)^{k}+\mu\right] \pi_{\mathrm{obs}}^{\mathrm{soc}}(k) } & =\mu \pi_{\mathrm{obs}}^{\mathrm{soc}}(k+1)+\lambda(1-\theta)^{k-1} \pi_{\mathrm{obs}}^{\mathrm{soc}}(k-1), \quad 1 \leq k \leq n_{s}, \\
\mu \pi_{\mathrm{obs}}^{\mathrm{soc}}\left(n_{s}+1\right) & =\lambda(1-\theta)^{n_{s}} \pi_{\mathrm{obs}}^{\mathrm{soc}}\left(n_{s}\right) .
\end{aligned}
$$


Solving equations (8)-(10), we have

$$
\begin{aligned}
& \pi_{\mathrm{obs}}^{\mathrm{soc}}(n)=\pi_{\mathrm{obs}}^{\mathrm{soc}}(0)\left(\frac{\lambda}{\mu}\right)^{n}(1-\theta)^{n(n-1) / 2}, \quad 1 \leq n \leq n_{s}+1 \\
& \pi_{\mathrm{obs}}^{\mathrm{soc}}(0)=\left(\sum_{n=0}^{n_{s}+1}\left(\frac{\lambda}{\mu}\right)^{n}(1-\theta)^{n(n-1) / 2}\right)^{-1}
\end{aligned}
$$

Based on equations (11) and (12), $\sum_{k=0}^{n_{s}+1} k \pi_{\mathrm{obs}}^{\mathrm{soc}}(k)$ is used to get the expected number of groups in the team queue. Next, we use Little's formula, i.e., $E\left[L_{\text {obs }}\right]=\lambda\left(n_{s}\right) E[W]$, to obtain the expression of $E\left[L_{\mathrm{obs}}\right]$ :

$$
E\left[L_{\mathrm{obs}}\right]=\lambda\left(1-\pi_{\mathrm{obs}}^{\mathrm{soc}}\left(n_{s}+1\right)\right) \sum_{n=0}^{n_{s}} \pi_{\mathrm{obs}}^{\mathrm{soc}}(n) E\left[W_{n}\right]=\lambda\left(1-\pi_{\mathrm{obs}}^{\mathrm{soc}}\left(n_{s}+1\right)\right) \sum_{n=0}^{n_{s}} \pi_{\mathrm{obs}}^{\mathrm{soc}}(n) \frac{1-(1-\theta)^{n+1}}{\mu \theta}
$$

where $\lambda\left(n_{s}\right)$ records the effective arrival rate of the system in the observable case and $E[W]$ records the mean sojourn time of an arbitrary arrival who would join the team queue. If all new arrivals adopt a threshold strategy to join the team queue, then we could derive the expected social benefit by substituting equation (13) into $S_{\text {obs }}^{\text {soc }}\left(n_{s}\right)=\lambda\left(n_{s}\right) R-C E\left[L_{\text {obs }}\right]$.

From equation (6), it is found that it would be difficult to derive an analytical solution $n_{\text {soc }}$ to the optimal value that maximizes the expected social benefit. However, it can be easily computed through numerical algorithm. Next, we present the calculation process of Algorithm 1, and the numerical results would be shown in the Numerical Examples section.

\section{The Unobservable Case Analysis}

Now, we look at the unobservable team queue. In this case, new arrivals have no way of knowing how many groups are in the team queue and cannot observe in advance if they have friends in the queue. Since customers could know the value of parameter $\theta$, even if the queue is not observable, they could estimate the probability of joining (or creating) a group; that is, they could be assigned to each group with probability. According to the steady-state analysis, we then obtain their expected sojourn time and their strategic decisions on the basis of their expected utility. Hereby, we first give a lemma to express the expected utility of a joining customer.

Lemma 2. For the unobservable team queue, a new arrival does not know the state of the system; if all the other customers join the queue with probability $q$ and the new arrival choose to join the queue, then his utility is shown as follows:

$$
U_{\text {un }}(q)=\left(R-\frac{C}{\mu \theta}\right)+\frac{C(1-\theta)\left(1-\pi_{0}\right)}{\lambda q \theta},
$$

where $\pi_{0}$ satisfies

$$
\pi_{0}=\left(\sum_{n=0}^{\infty}\left(\frac{\lambda q}{\mu}\right)^{n}(1-\theta)^{n(n-1) / 2}\right)^{-1}
$$

Proof. First, let $\pi_{n}, n \in \Omega$ record the steady-state distribution of the team queue under the unobservable case. Suppose that all new arrivals join the team queue with probability $q$; then, the balance equations of the team queue have the following forms:

$$
\begin{aligned}
\lambda q \pi_{0} & =\mu \pi_{1}, \\
{\left[\mu+\lambda q(1-\theta)^{n}\right] \pi_{n} } & =\lambda q(1-\theta)^{n-1} \pi_{n-1}+\mu \pi_{n+1}, \quad n \geq 1 .
\end{aligned}
$$

Combining with the normalization condition $\sum_{n=0}^{\infty} \pi_{n}=1$, we have

$$
\begin{aligned}
& \pi_{n}=\pi_{0}\left(\frac{\lambda q}{\mu}\right)^{n}(1-\theta)^{n(n-1) / 2}, \quad n \geq 1 \\
& \pi_{0}=\left(\sum_{n=0}^{\infty}\left(\frac{\lambda q}{\mu}\right)^{n}(1-\theta)^{n(n-1) / 2}\right)^{-1}
\end{aligned}
$$

Since $\pi_{0}$ is finite, the system is stable if $0<\theta \leq 1$. In particular, the stability condition of the system is $\lambda q<\mu$ if $\theta=0$. Define $\Pi(z)=\sum_{n=0}^{\infty} \pi_{n} z^{n}$; multiplying both sides of equation (17) by $z^{n}$ and summing all the terms, we have

$$
\Pi(z)=\frac{\lambda q z}{\mu} \Pi((1-\theta) z)+\pi_{0} .
$$

Substituting $z=1$ into $\Pi(z)$ and combining $\Pi(1)=1$, we have

$$
\Pi(1-\theta)=\frac{\mu}{\lambda q}\left(1-\pi_{0}\right) .
$$

Then, for the unobservable case, if all the other customers join the queue with probability $q$, the expected utility of a new arrival is 


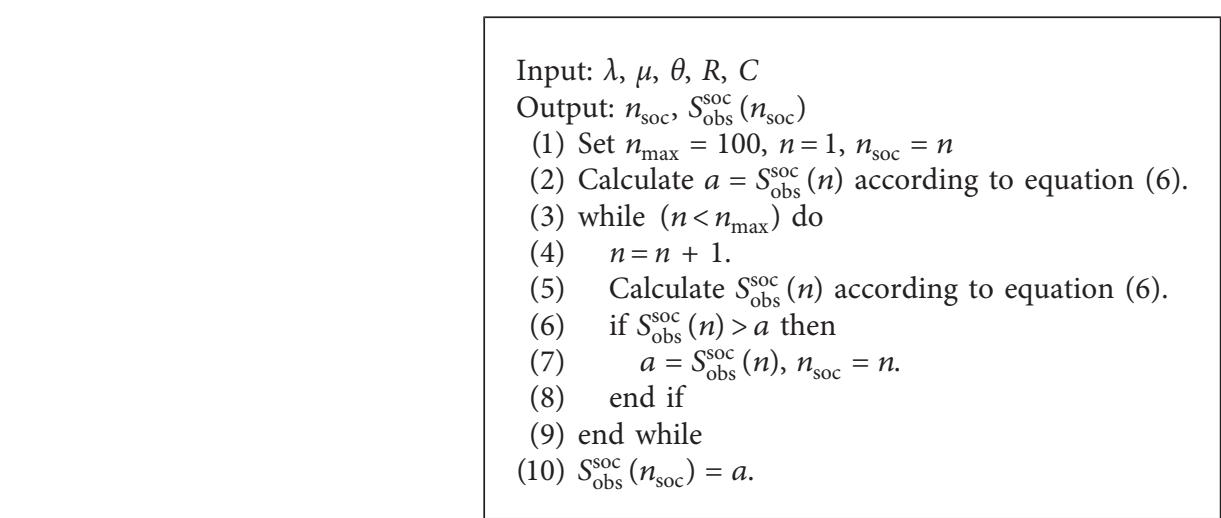

Algorithm 1: Search for $n_{\text {soc }}$ and $S_{\text {obs }}^{\text {soc }}\left(n_{\text {soc }}\right)$.

$U_{\mathrm{un}}(q)=\sum_{n=0}^{\infty} \pi_{n} U_{\mathrm{obs}}(n)=\left(R-\frac{C}{\mu \theta}\right)+\frac{C(1-\theta)\left(1-\pi_{0}\right)}{\lambda q \theta}$

which is equation (14).

We can now decide the equilibrium joining strategies of an arriving customer in the unobservable case based on the above discussion. The following theorem includes the explicit results.

Theorem 3. For the unobservable model, there is a unique mixed strategy $q_{e}$, which takes the form of

$$
q_{e}= \begin{cases}0, & R \leq \frac{C}{\mu} \\ q_{e}^{*}, \quad \frac{C}{\mu}<R<\frac{C}{\mu \theta}-\frac{C(1-\theta)\left(1-\pi_{0}(1)\right)}{\lambda \theta} \\ 1, \quad R \geq \frac{C}{\mu \theta}-\frac{C(1-\theta) \pi_{0}(1)}{\lambda \theta}\end{cases}
$$

where $q_{e}^{*}$ is the solution which satisfies

$$
\frac{1-\pi_{0}(q)}{q}=\frac{\lambda}{\mu(1-\theta)}-\frac{\lambda R \theta}{C(1-\theta)} .
$$

Proof. Hence, $\pi_{0}$ is a function depends on $q$. Define $\pi_{0}(q)=(1 / A(q))$, where $A(q)=\sum_{n=0}^{\infty}(\lambda q / \mu)^{n}$ $(1-\theta)^{n(n-1) / 2}$. Then, we have

$$
\pi_{0}^{\prime}(q)=-\frac{A^{\prime}(q)}{[A(q)]^{2}}=-\frac{\sum_{n=1}^{\infty} n(\lambda / \mu)(\lambda q / \mu)^{n-1}(1-\theta)^{n(n-1) / 2}}{[A(q)]^{2}} .
$$

Differentiating $U_{\text {un }}(q)$, we then have

$$
U_{\mathrm{un}}^{\prime}(q)=-\frac{C(1-\theta)}{\lambda \theta} \frac{\left(\sum_{n=0}^{\infty}(\lambda q / \mu)^{n}(1-\theta)^{n(n-1) / 2}\right)^{2}-\sum_{n=0}^{\infty}(n+1)(\lambda q / \mu)^{n}(1-\theta)^{n(n-1) / 2}}{q^{2}(A(q))^{2}} .
$$

It is not difficult to find that

$$
\left(\sum_{n=0}^{\infty}\left(\frac{\lambda q}{\mu}\right)^{n}(1-\theta)^{n(n-1) / 2}\right)^{2}-\sum_{n=0}^{\infty}(n+1)\left(\frac{\lambda q}{\mu}\right)^{n}(1-\theta)^{n(n-1) / 2}>0
$$


So, we have $U_{\text {un }}^{\prime}(q)<0$. This means that $U_{\text {un }}(q)$ is monotone decreasing with respect to $q$. Next, the issue can be solved by considering the following three scenarios:

Scenario 1. If $U_{\text {un }}(0) \leq 0$, we have $R \leq(C / \mu)$, then $U_{\text {un }}(q)$ is a nonpositive function for every $q$, balking is the unique equilibrium strategy, and we can obtain the first branch of equation (23).

Scenario 2. If $U_{\text {un }}(0)>0$ and $U_{\text {un }}(1)<0$, we have $(C / \mu)<R<(C / \mu \theta)-\left(C(1-\theta)\left(1-\pi_{0}(1)\right) / \lambda \theta\right)$, there is a unique root $q_{e}^{*}$ in the open interval $(0,1)$ that satisfies $U_{\text {un }}\left(q_{e}^{*}\right)=0$, and then we can obtain the second branch of equation (23).

Scenario 3. If $U_{\text {un }}(1) \geq 0$, we have $R \geq(C / \mu \theta)-\left(C(1-\theta)\left(1-\pi_{0}(1)\right) / \lambda \theta\right)$, then $U_{\text {un }}(q)$ is a nonnegative function for every $q$; in this scenario, joining is the unique equilibrium strategy and we could obtain the third branch of equation (23).

The expected social welfare for the unobservable case is then considered. The following theorem can be used to obtain the explicit result.

Theorem 4. For the unobservable model, if all arrivals adopt a mixed strategy with joining probability $q$, then the expected net benefit is

$$
S_{\mathrm{un}}^{\mathrm{soc}}(q)=\lambda q R-C\left(\frac{\lambda q}{\mu \theta}-\frac{(1-\theta)\left(1-\pi_{0}(q)\right)}{\theta}\right),
$$

where $\pi_{0}(q)$ is obtain in equation (19).

Proof. If all new arrivals join the queue by adopting a mixed strategy, then the expected net benefit is

$$
S_{\mathrm{un}}^{\mathrm{soc}}(q)=\lambda q R-\operatorname{CE}\left[L_{\mathrm{un}}\right],
$$

where $E\left[L_{\text {un }}\right]$ is obtained by Little's formula; i.e., combining equation (14) and using Little's formula, we have

$$
E\left[L_{\text {un }}\right]=\left(\frac{\lambda q}{\mu \theta}-\frac{(1-\theta)\left(1-\pi_{0}(q)\right)}{\theta}\right) .
$$

Substituting $E\left[L_{\text {un }}\right]$ into $S_{\text {un }}^{\text {soc }}(q)=\lambda q R-C E\left[L_{\text {un }}\right]$, we then have the expression of equation (28).

From the expression of $\pi_{0}(q)$, we find that it is difficult to obtain the optimal value $q_{\mathrm{soc}}$ with explicit analytical form. However, $q_{\text {soc }}$ is easily obtained by numerical calculation. Hence, we will present numerical Algorithm 2 and give some numerical examples in the following section to derive some qualitative conclusions.

\section{Numerical Examples}

In this section, by using Matlab software, we would display the dependence of the equilibrium strategies and social optimal strategies on parameters through some numerical examples. Assuming that $\lambda=1.5, \mu=2$, and $\theta=0.1$, the effect of $R$ and $C$ on the individual and social optimal thresholds are studied, respectively. Obviously, in Figures 1 and 2, both of the values $n_{e}$ and $n_{\text {soc }}$ increase as $R$ increases and decrease as $C$ increases. It makes sense that queueing customers would be willing to join the queue if they were offered with a higher value reward. Conversely, if the cost of delay is high, queueing customers tend to balk to avoid paying too much.

Next, we continue to suppose that $\lambda=1.5, \mu=2$, and $\theta=0.1$ and observe the changing relationship between dependent variables $\left(q_{e}\right.$ and $\left.q_{\mathrm{soc}}\right)$ and independent variables $(R$ and $C)$. Obviously, in Figure $3, q_{e}$ and $q_{\text {soc }}$ increase as $R$ increases. The reason is that, as $R$ increases, queueing customers could perceive higher service value and prefer to join the team queue. In Figure 4 , it is obvious that $q_{e}$ and $q_{\text {soc }}$ decrease as $C$ increases. In order to find how the parameters $\lambda$ and $\mu$ affect $q_{e}$ and $q_{\mathrm{soc}}$, we then assume $\theta=0.1, C=2$, and $R=4$ and show their relationship in Figures 5 and 6. From Figures 5 and 6, we observe that $q_{e}$ and $q_{\mathrm{soc}}$ are continuously increasing with respect to $\mu$ and continuously decreasing with respect to $\lambda$. This is because that increasing the service rate can benefit customers by reducing the expected delay cost and, meanwhile, the new arriving customers who know a high arrival rate can predict the higher load of the system, which increases their delay cost and makes them reluctant to join the system.

Finally, in Figures 1 and 2, we note that the inequality $n_{\text {soc }} \leq n_{e}$ holds for any values of $R$ and $C$, and in Figures 3 and 4 , we note that the inequality $q_{\text {soc }} \leq q_{e}$ holds for any values of $R$ and $C$. Moreover, in Figures 5 and 6 , the inequality $q_{\mathrm{soc}} \leq q_{e}$ holds for any values of $\lambda$ and $\mu$. The main reason for these results is that each new arrival is inclined to maximizing his/her own benefit. When new arrivals join the team queue, their joining decisions would lengthen the waiting time and increase the delay cost of future arrivals; that is, it will have a negative externality effect on future arrivals. Therefore, when maximizing the net benefit, we should consider these negative externalities, which will directly result in the inequalities $n_{\mathrm{soc}} \leq n_{e}$ and $q_{\mathrm{soc}} \leq q_{e}$. Furthermore, we also find that the inequalities are in accordance with the results in some classic papers: individual optimization results in longer queue than it is socially desirable. We expect the obtained results can be extended to other practice queueing systems.

From the above numerical results, we could obtain the following management inspiration: First, the higher the service level is, the more will be the customers joining the queue. The service provider should effectively improve service quality at a certain cost. Second, customers' perceived wait time periods will be longer in a boring and monotonous environment. High delay sensitivity and long perceived wait time periods make customers balk the queue; therefore, the service provider should take measures and use the theory of queueing psychology to 
Input: $\lambda, \mu, \theta, R, C$

Output: $q_{\mathrm{soc}}, S_{\mathrm{un}}^{\mathrm{soc}}\left(q_{\mathrm{soc}}\right)$

(1) Set step $=0.01, q=$ step, $q_{\text {un }}=q$

(2) Calculate $a=S_{\text {un }}^{\text {soc }}(q)$ according to equation (28).

(3) while $(q<1)$ do

(4) $q=q+$ step.

(5) Calculate $S_{\text {un }}^{\text {soc }}(q)$ according to equation (28).

(6) if $S_{\text {un }}^{\mathrm{soc}}(q)>a$ then

(7) $\quad a=S_{\mathrm{un}}^{\mathrm{soc}}(q), q_{\mathrm{soc}}=q$.

(8) end if

(9) end while

(10) $S_{\text {un }}^{\text {soc }}\left(q_{\text {soc }}\right)=a$.

Algorithm 2: Search for $q_{\mathrm{soc}}$ and $S_{\mathrm{un}}^{\mathrm{soc}}\left(q_{\mathrm{soc}}\right)$.

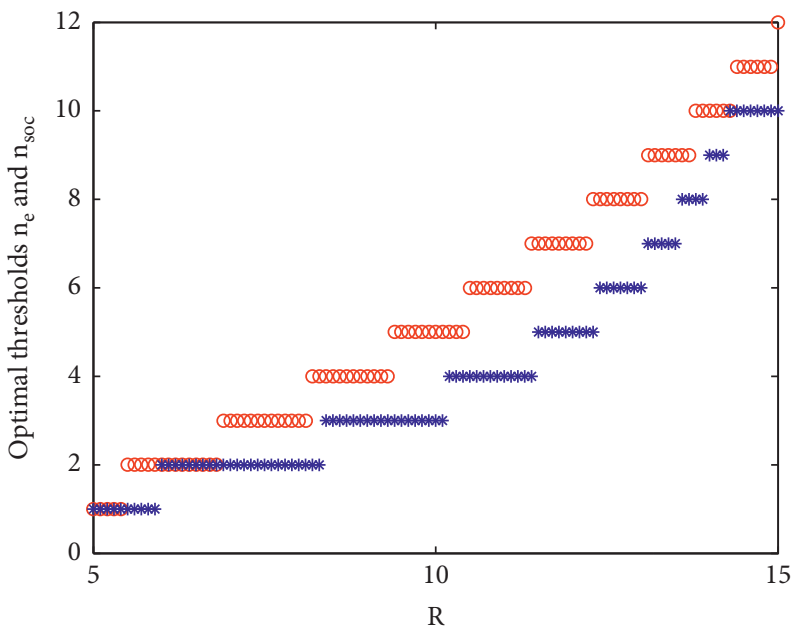

- Equilibrium threshold $n_{e}$

* Social optimal threshold $\mathrm{n}_{\text {soc }}$

Figure $1: n_{e}$ and $n_{\text {soc }}$ vs $R(C=4)$.

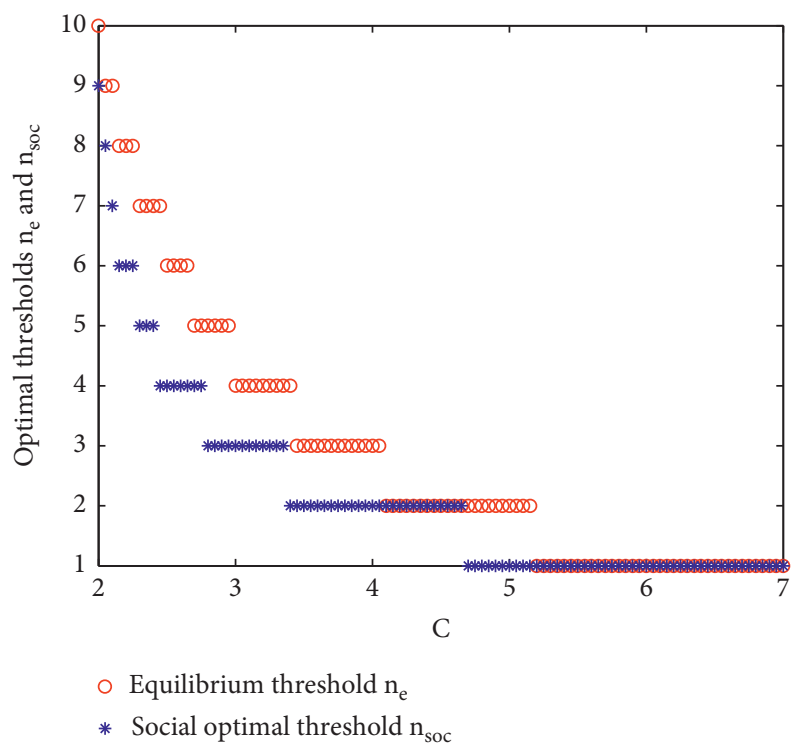

Figure $2: n_{e}$ and $n_{\text {soc }}$ vs $C(R=7)$. 


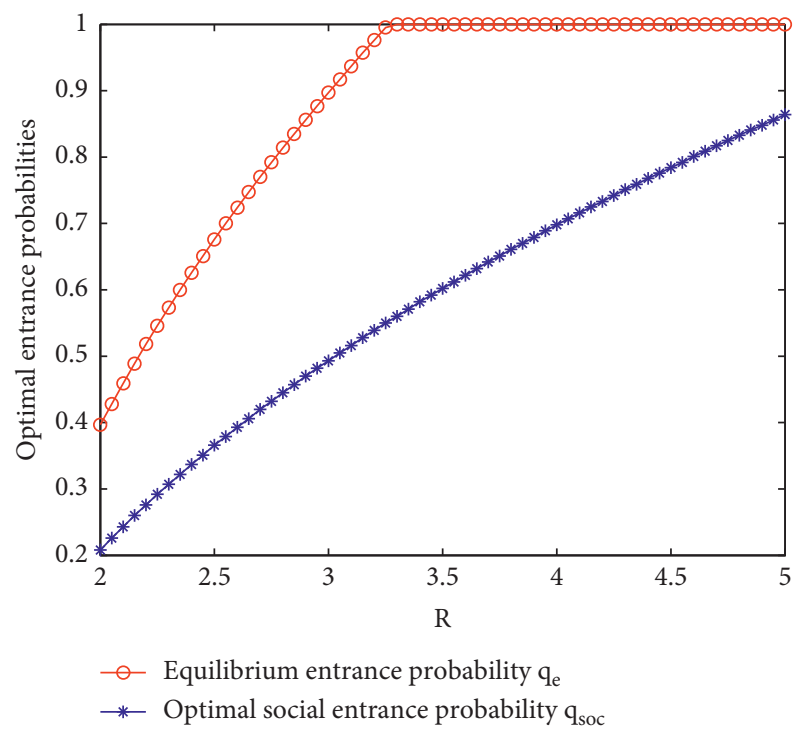

Figure 3: $q_{e}$ and $q_{\text {soc }}$ vs $R(C=3)$.

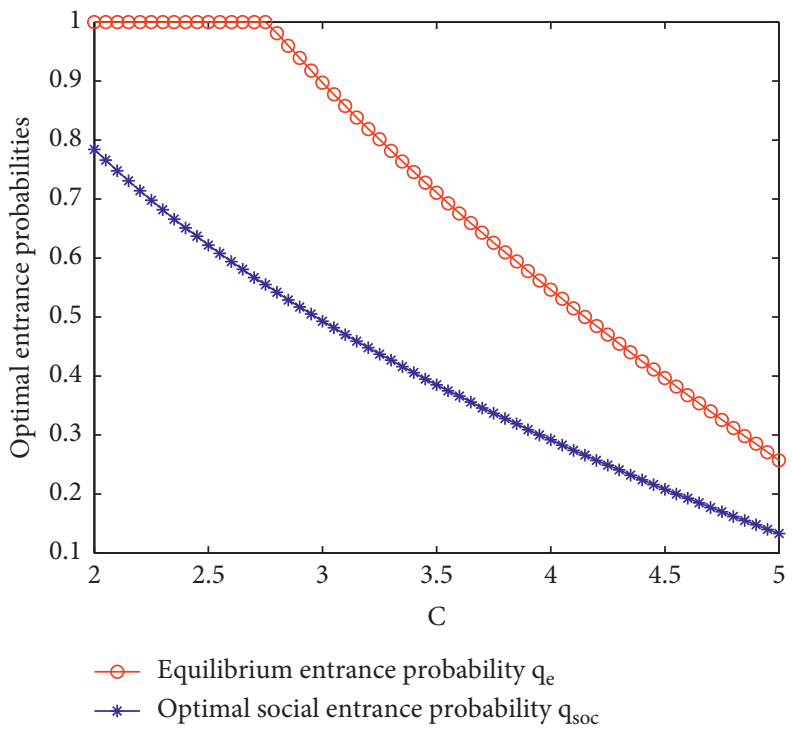

Figure $4: q_{e}$ and $q_{\text {soc }}$ vs $C(R=3)$. 


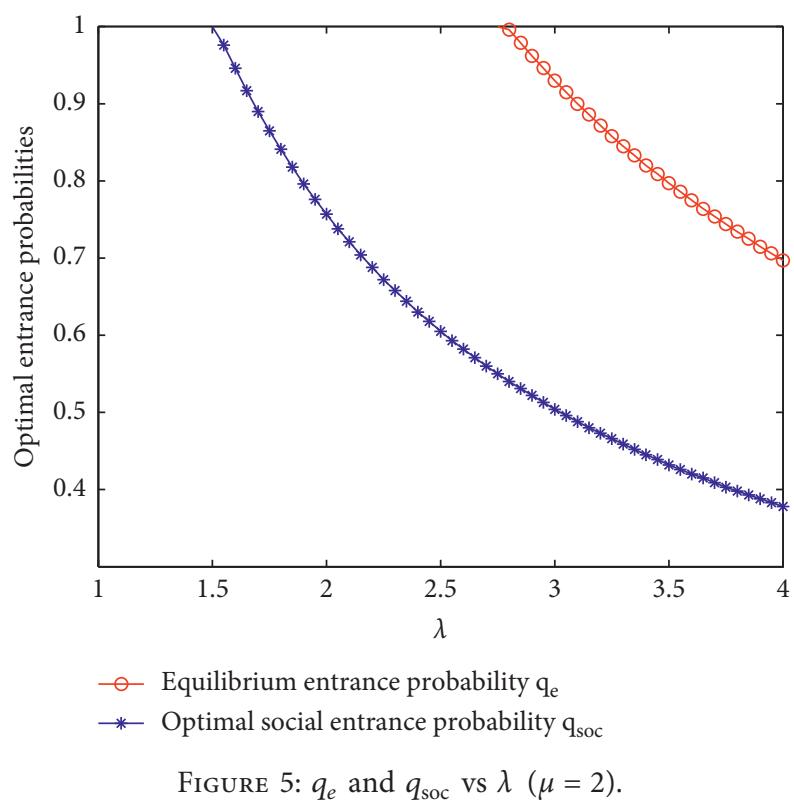

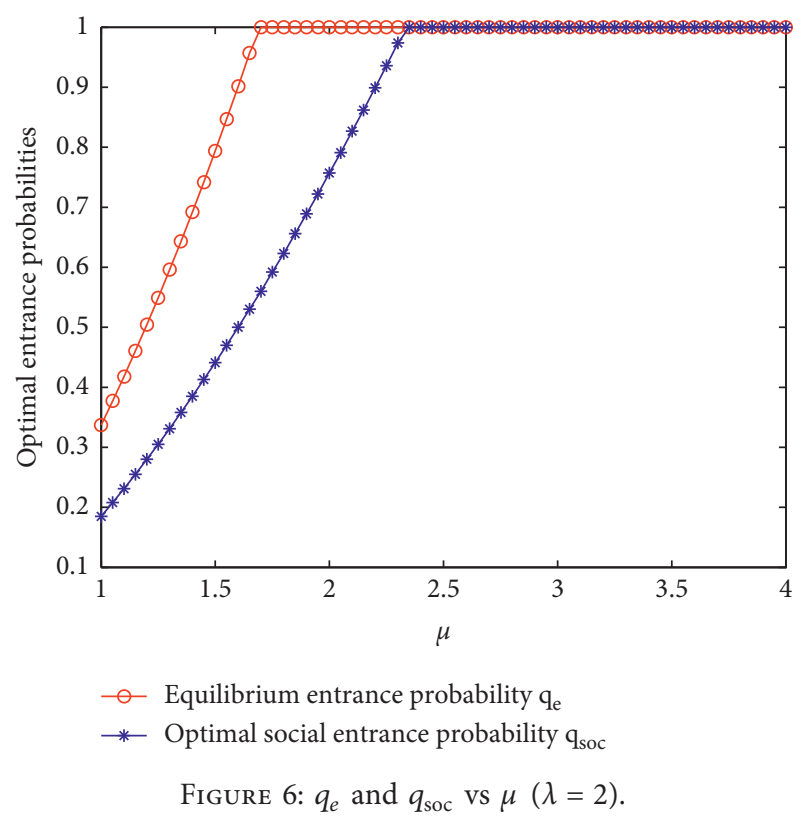

reduce customers' perceived waiting time (by reducing the delay sensitivity) without shortening the actual waiting time, such as "improve the queueing situation," "fill the waiting time," and "make customers feel that the service has started."

\section{Summary and Conclusions}

In this paper, the strategic behavior of queueing customers in the ticket windows at scenic spots is analyzed. We assumed that each arriving customer first finds his friend in the queue so as to cut in the queue and complete his corresponding service as early as possible. Therefore, the underlying queueing service phenomenon can be modelled as a "team queue." For the observable case, we first derived the individual optimal threshold strategies and the expected net benefit. Then, for the unobservable case, the mixed equilibrium balking strategies and the social optimal strategies are obtained. Finally, a number of numerical examples are given to compare the equilibrium strategy with the social optimal strategy. In the future work, by using the real data, we could investigate a real-world ticket window at scenic spot and verify the validity of the derived conclusions; meanwhile, we could also investigate the pricing of service at scenic spots and the effect of information on queueing customers' strategic behavior. We think these would be interesting directions for future research.

\section{Data Availability}

All data included in this study are available upon request to the corresponding author.

\section{Conflicts of Interest}

The authors declare that there are no conflicts of interest regarding the publication of this paper.

\section{Acknowledgments}

This work was supported by the National Natural Science Foundation of China (Grant no. 12001329) and Scientific Research Foundation of Shandong University of Science and Technology for Recruited Talent (Grant no. 2019RCJJ016).

\section{References}

[1] Q.-M. He and A. A. Chavoushi, "Analysis of queueing systems with customer interjections," Queueing Systems, vol. 73, no. 1, pp. 79-104, 2013.

[2] O. Boxma, J. V. D. Wal, and U. Yechiali, "Polling with batch service," Stochastic Models, vol. 24, no. 4, pp. 604-625, 2008. 
[3] N. Perel and U. Yechiali, "The Israeli queue with infinite number of groups," Probability in the Engineering and Informational Sciences, vol. 28, no. 1, pp. 1-19, 2014.

[4] N. Perel and U. Yechiali, "The Israeli queue with priorities," Stochastic Models, vol. 29, no. 3, pp. 353-379, 2013.

[5] N. Perel and U. Yechiali, "The Israeli Queue with retrials," Queueing Systems, vol. 78, no. 1, pp. 31-56, 2014.

[6] N. Perel and U. Yechiali, "The Israeli Queue with a general group-joining policy," Annals of Operations Research, 2015.

[7] R. C. Larson, "OR forum-perspectives on queues: social justice and the psychology of queueing," Operations Research, vol. 35, no. 6, pp. 895-905, 1987.

[8] P. Afèche and H. Mendelson, "Pricing and priority auctions in queueing systems with a generalized delay cost structure," Management Science, vol. 50, pp. 869-882, 2004.

[9] T. Jiang, L. Liu, and L. Liu, "Analysis of a batch service multiserver polling system with dynamic service control," Journal of Industrial \& Management Optimization, vol. 14, no. 2, pp. 743-757, 2018.

[10] T. Jiang, L. Liu, and Y. Zhu, "Analysis of a batch service polling system in a multi-phase random environment," Methodology and Computing in Applied Probability, vol. 20, no. 2, pp. 699-718, 2018.

[11] T. Jiang, "Tail asymptotics for a batch service polling system with retrials and nonpersistent customers," Journal of Mathematical Analysis and Applications, vol. 459, no. 2, pp. 893-905, 2018.

[12] P. Naor, "The regulation of queue size by levying tolls," Econometrica, vol. 37, no. 1, pp. 15-24, 1969.

[13] N. M. Edelson and D. K. Hilderbrand, "Congestion tolls for Poisson queuing processes," Econometrica, vol. 43, no. 1, pp. 81-92, 1975.

[14] R. Hassin and M. Haviv, To Queue or Not to Queue: Equilibrium Behavior in Queueing Systems, Kluwer Academic Publishers, Boston, MA, USA, 2003.

[15] R. Hassin, Rational Queueing, CRC Press, Taylor and Francis Group, Boca Raton, FL, USA, 2016.

[16] R. Hassin and R. Roet-Green, "The impact of inspection cost on equilibrium, revenue, and social welfare in a single-server queue," Operations Research, vol. 65, no. 3, pp. 804-820, 2017.

[17] R. Ibrahim, "Sharing delay information in service systems: a literature survey," Queueing Systems, vol. 89, no. 1-2, pp. 49-79, 2018.

[18] A. Economou and A. Manou, "Equilibrium balking strategies for a clearing queueing system in alternating environment," Annals of Operations Research, vol. 208, no. 1, pp. 489-514, 2013.

[19] A. Manou, A. Economou, and F. Karaesmen, "Strategic customers in a transportation station: when is it optimal to wait?” Operations Research, vol. 62, no. 4, pp. 910-925, 2014.

[20] O. Bountali and A. Economou, "Equilibrium joining strategies in batch service queueing systems," European Journal of Operational Research, vol. 260, no. 3, pp. 1142-1151, 2017.

[21] O. Bountali and A. Economou, "Equilibrium threshold joining strategies in partially observable batch service queueing systems," Annals of Operations Research, vol. 277, no. 2, pp. 231-253, 2019.

[22] Z. Wang, L. Liu, Y. Shao, X. Chai, and B. Chang, "Equilibrium joining strategy in a batch transfer queuing system with gated policy," Methodology and Computing in Applied Probability, vol. 22, no. 1, pp. 75-99, 2020.

[23] H. Afimeimounga, W. Solomon, and I. Ziedins, "User equilibria for a parallel queueing system with state dependent routing," Queueing Systems, vol. 66, no. 2, pp. 169-193, 2010.
[24] Y. Chen, M. Holmes, and I. Ziedins, "Monotonicity properties of user equilibrium policies for parallel batch systems," Queueing Systems, vol. 70, no. 1, pp. 81-103, 2012. 